PROCEEDINGS OF THE

AMERICAN MATHEMATICAL SOCIETY

Volume 127, Number 8, Pages 2423-2429

S 0002-9939(99)04794-2

Article electronically published on March 23, 1999

\title{
BROWNIAN SPACE-TIME FUNCTIONS OF ZERO QUADRATIC VARIATION DEPEND ONLY ON TIME
}

\author{
P. J. FITZSIMMONS
}

(Communicated by Stanley Sawyer)

\begin{abstract}
Let $B_{t}, t \geq 0$, be a 1-dimensional Brownian motion and let $f: \mathbb{R} \times$ $\left[0, \infty\left[\rightarrow \mathbb{R}\right.\right.$ be a continuous function. We show that if $t \mapsto f\left(B_{t}, t\right)$ is locally of zero quadratic variation, then $f(x, t)=f(0, t)$ for all $(x, t) \in \mathbb{R} \times[0, \infty[$. This result extends recent work of $\mathrm{F}$. B. Knight, thereby confirming a conjecture of T. Salisbury.
\end{abstract}

\section{INTRODUCTION}

Let $\left(B_{t}\right)_{t \geq 0}$ be standard 1-dimensional Brownian motion with $B_{0}=0$, let $f: \mathbb{R} \times$ $[0, \infty) \rightarrow \mathbb{R}$ be a continuous function, and let

$$
V(f):=\sup \sum_{i}\left|f\left(B_{t_{i+1}}, t_{i+1}\right)-f\left(B_{t_{i}}, t_{i}\right)\right|
$$

(the supremum extending over all finite partitions of $[0,1]$ ) denote the total variation of the process $f\left(B_{t}, t\right), 0 \leq t \leq 1$. In a recent paper [5], F. B. Knight discussed a problem raised by T. S. Salisbury: Show that if $V(f)$ is finite almost surely, then $f$ is a function of $t$ alone. Knight proved that if the expectation of $V(f)$ is finite, then $f$ is indeed a function of its second variable only. In this note we shall prove the following result, which contains the solution of Salisbury's problem as a special case.

Theorem 1. Let $f: \mathbb{R} \times[0, \infty) \rightarrow \mathbb{R}$ be a continuous function, and suppose that $t \mapsto f\left(B_{t}, t\right)$ is locally of zero quadratic variation, in the sense that for all $T>0$,

$$
\lim _{n \rightarrow \infty} \sum_{i=0}^{\left\lfloor 2^{n} T\right\rfloor-1}\left[f\left(B_{(i+1) 2^{-n}},(i+1) 2^{-n}\right)-f\left(B_{i 2^{-n}}, i 2^{-n}\right)\right]^{2}=0
$$

in probability. Then $f(x, t)=f(0, t)$ for all $x \in \mathbb{R}$ and all $t \geq 0$.

Notice that if the total variation of a continuous function $g:[0, \infty[\rightarrow \mathbb{R}$ is finite on some interval $[0, T]$, then its quadratic variation (over the dyadic rationals, as in $(1))$ is zero on $[0, T]$.

Salisbury's problem arose in connection with the following question: Must a continuous strong Markov process with paths of bounded variation (in $\mathbb{R}^{d}$ ) be a

Received by the editors September 8, 1997 and, in revised form, October 24, 1997.

1991 Mathematics Subject Classification. Primary 60J65; Secondary 60J55.

Key words and phrases. Brownian motion, quadratic variation, time reversal, Girsanov transformation. 
deterministic function of its starting point? According to unpublished work of E. Çinlar and J. Jacod, the answer is 'Yes' when $d=1$. A striking counterexample for $d \geq 2$ has been provided by Salisbury in [7].

A very short solution of Salisbury's problem is available in the time-independent case. Suppose that $f: \mathbb{R} \rightarrow \mathbb{R}$ is a continuous function such that the total variation of $t \mapsto f\left(B_{t}\right)$ on $[0,1]$ is finite almost surely. By a theorem of Banach, if $g:[0,1] \rightarrow \mathbb{R}$ is a continuous function, then its total variation is given by the integral

$$
\int_{-\infty}^{\infty} N(y) d y
$$

where $N(y)$ is the cardinality of the level set $\{t \in[0,1]: g(t)=y\}$. Taking $g(t)=f\left(B_{t}(\omega)\right)$ for a fixed sample point $\omega$, we see that for almost every pair $(\omega, y)$ the level set $\left\{t \in[0,1]: B_{t}(\omega) \in f^{-1}(\{y\})\right\}$ is finite. But if $H$ is a non-empty Borel subset of $\mathbb{R}$, then the level set $\left\{t \in[0,1]: B_{t}(\omega) \in H\right\}$ is uncountable for all $\omega$ in a set of positive probability. It follows that $f^{-1}(\{y\})$ is empty for (Lebesgue) almost every $y \in \mathbb{R}$, so $f$ is constant by the Intermediate Value Theorem.

The argument just given is quite robust - it is valid for any irreducible continuous-path strong Markov process that hits a given closed set infinitely often, if at all. However, even the time-independent case of Theorem 1 appears to be out of reach of this method.

The reader will have verified by now that if $f$ is smooth enough to allow the application of Itô's formula, then the hypothesis of Theorem 1 implies that the local martingale $\int_{0}^{t} \frac{\partial f}{\partial x}\left(B_{s}, s\right) d B_{s}$ is of zero quadratic variation. That is, $\int_{0}^{t}\left[\frac{\partial f}{\partial x}\left(B_{s}, s\right)\right]^{2} d s \equiv$ 0 almost surely, from which it follows easily that $\partial f / \partial x$ vanishes. Knight's approach to the problem involved a reduction of the general case to the smooth case by mollification.

Our proof of Theorem 1 is based (in a different way) on an extension of Itô's formula due to Föllmer, Protter and Shiryaev [3]. Briefly stated, the argument is as follows. Consider the generator of space-time Brownian motion with drift $f$; namely, $L=\frac{1}{2} \partial^{2} / \partial x^{2}+\partial / \partial t+f(x, t) \partial / \partial x$. The formal adjoint of $L$ is the operator $L^{*}:=\frac{1}{2} \partial^{2} / \partial x^{2}-\partial / \partial t-f(x, t) \partial / \partial x+[\partial f / \partial x]$, whereas the informal time reversal of the process associated with $L$ has generator $\hat{L}:=\frac{1}{2} \partial^{2} / \partial x^{2}-\partial / \partial t-f(x, t) \partial / \partial x$, as we show using results from [3]. Because $L^{*}$ and $\hat{L}$ agree on smooth functions of compact support, the distribution-sense partial derivative $\partial f / \partial x$ must vanish.

Theorem 1 will be proved in the next section. In a final section we indicate several related results.

\section{Proof of Theorem 1}

We assume that $B=\left(B_{t}\right)_{t \geq 0}$ is the coordinate process $B_{t}(\omega)=\omega(t)$ on the space $\Omega$ of continuous paths $\omega:\left[0, \infty\left[\rightarrow \mathbb{R}\right.\right.$. We write $\left(\mathcal{F}_{t}\right)_{t \geq 0}$ for the natural filtration of $B$, and $P^{x}$ for the law of $B$ under the initial condition $B_{0}=x$.

There is no loss of generality in assuming $f$ is bounded. For if $f_{N}(x, t):=$ $f(x, t) \vee N \wedge(-N)$, then the quadratic variation of $f_{N}\left(B_{t}, t\right)$ is no larger than that of $f\left(B_{t}, t\right)$. Once the theorem is proved for bounded functions, a passage to the limit will secure the general case. 
Let $X=\left(X_{t}\right)_{t \geq 0}$ be the forward space-time process defined on the sample space $\bar{\Omega}:=\Omega \times] 0, \infty[$ by

$$
X_{t}(\omega, u)=\left(B_{t}(\omega), u+t\right), \quad \omega \in \Omega, u>0, t \geq 0 .
$$

The state space of $X$ is $E:=\mathbb{R} \times] 0, \infty[$, and it is easy to check that $X$ is in duality (with respect to Lebesgue measure on $E$ ) with the backward space-time process $\hat{X}=\left(\hat{X}_{t}\right)_{t \geq 0}$ defined on $\bar{\Omega}$ by

$$
\hat{X}_{t}(\omega, u)= \begin{cases}\left(B_{t}(\omega), u-t\right) & 0 \leq t<u \\ \delta & t \geq u\end{cases}
$$

Here $\delta$ is a cemetery state adjoined to $E$ as an isolated point. Both $X$ and $\hat{X}$ are strong Markov processes under the laws $P^{x, u}:=P^{x} \otimes \epsilon_{u}$ on $\bar{\Omega}$.

We now use the function $f$ to perform drift transformations of $X$ and $\hat{X}$, thereby obtaining a second pair of space-time processes. Since $f$ is bounded, the formula

$$
Z_{t}(\omega, u):=\exp \left(\int_{0}^{t} f\left(B_{s}, u+s\right) d B_{s}-\frac{1}{2} \int_{0}^{t} f\left(B_{s}, u+s\right)^{2} d s\right)
$$

defines a positive continuous martingale of $X$ under any of the laws $P^{x, u},(x, u) \in E$. Define new probability laws $Q^{x, u}$ on $\bar{\Omega}$ by

$$
Q^{x, u}(A):=P^{x, u}\left(Z_{t} ; A\right), \quad A \in \mathcal{G}_{t}, t \geq 0,
$$

where $\left(\mathcal{G}_{t}\right)_{t \geq 0}$ is the filtration generated by $X$. By general theory [8, 662$], X$ under the laws $\left\{Q^{x, u}:(x, u) \in E\right\}$ is a time-homogeneous strong Markov process with continuous paths. Moreover, by the Cameron-Martin-Girsanov theorem, the associated $L^{2}$ infinitesimal generator is given by

$$
L \varphi(x, u):=\frac{1}{2} \frac{\partial^{2} \varphi}{\partial x^{2}}(x, u)+\frac{\partial \varphi}{\partial u}(x, u)+f(x, u) \frac{\partial \varphi}{\partial x}(x, u),
$$

provided $\varphi$ is smooth and compactly supported in $E$. In fact, for such $\varphi$, Itô's formula implies that

$$
\varphi\left(X_{t}\right)-\int_{0}^{t}\left[\frac{1}{2} \frac{\partial^{2} \varphi}{\partial x^{2}}+\frac{\partial \varphi}{\partial u}\right]\left(X_{s}\right) d s
$$

is a continuous $P^{x, u}$-martingale. Thus, by $[6$, Thm. (1.7), p. 315], the process

$$
M_{t}:=\varphi\left(X_{t}\right)-\int_{0}^{t} L \varphi\left(X_{s}\right) d s
$$

is a continuous $Q^{x, u}$-local martingale. But $\varphi$ and $L \varphi$ are bounded and compactly supported in $E$, so $M$ is a $Q^{x, u}$-martingale, by [6, Prop. (1.7), p. 118]. From this it follows easily that $t^{-1}\left[Q^{x, u}\left(\varphi\left(X_{t}\right)\right)-\varphi(x, u)\right]$ converges to $L \varphi(x, u)$ in $L^{2}(E, d x \otimes d u)$.

We transform $\hat{X}$ similarly, using the density process

$$
\hat{Z}_{t}(\omega, u):=\exp \left(-\int_{0}^{t \wedge u} f\left(B_{s}, u-s\right) d B_{s}-\frac{1}{2} \int_{0}^{t \wedge u} f\left(B_{s}, u-s\right)^{2} d s\right),
$$

thereby obtaining a space-time diffusion with laws $\hat{Q}^{x, u}$ and $L^{2}$ infinitesimal generator

$$
\hat{L} \varphi(x, u)=\frac{1}{2} \frac{\partial^{2} \varphi}{\partial x^{2}}(x, u)-\frac{\partial \varphi}{\partial u}(x, u)-f(x, u) \frac{\partial \varphi}{\partial x}(x, u) .
$$


Notice that the lifetime of $\hat{X}$ is $u$ under $P^{x, u}$ or $\hat{Q}^{x, u}$.

Let us write $\left(Q_{t}\right)$ and $\left(\hat{Q}_{t}\right)$ for the transition semigroups corresponding to $Q^{x, u}$ and $\hat{Q}^{x, u}$, respectively. The crucial observation is that

$$
\left(\psi, Q_{t} \varphi\right)=\left(\hat{Q}_{t} \psi, \varphi\right)
$$

for all smooth compactly-supported functions $\psi$ and $\varphi$, where the pairing $(\cdot, \cdot \cdot)$ is the inner product in $L^{2}(E, d x \otimes d u)$. To see this we need to recall that if $P$ denotes $\int_{\mathbb{R}} P^{x} d x$ (the law of Brownian motion with Lebesgue measure as "initial distribution"), then, for each $t>0,\left.P\right|_{\mathcal{F}_{t}}$ is invariant with respect to the time reversal mapping $r_{t}$ defined on $\Omega$ by

$$
\left[r_{t} \omega\right](s):=\omega(t-s), \quad 0 \leq s \leq t .
$$

(Actually, $r_{t}$ is a mapping on equivalence classes of paths (agreeing on $[0, t]$ ); see $[10, \S 2]$.$) In addition, we require the following key fact, a slight extension of a result$ of Föllmer, Protter, and Shiryaev [3, Thm. 3.3, (2.19)].

Lemma 1. Let $g: \mathbb{R} \times[0, \infty[\rightarrow \mathbb{R}$ be a continuous function. Let

$$
D_{n}=\left\{0=t_{0}^{n}<t_{1}^{n}<\cdots<t_{k}^{n}<\cdots\right\}, \quad n \in \mathbb{N},
$$

be a nested sequence of locally finite partitions of $\left[0, \infty\left[\right.\right.$ with $\lim _{k} t_{k}^{n}=\infty$ for each $n$, and $\lim _{n} \sup _{k}\left|t_{k+1}^{n}-t_{k}^{n}\right|=0$.

(a) For each $x \in \mathbb{R}$ and $t>0$, the limit

$$
[g(B, \cdot), B]_{t}:=\lim _{n \rightarrow \infty} \sum_{t_{i}^{n} \leq t}\left[g\left(B_{t_{i+1}^{n}}, t_{i+1}^{n}\right)-g\left(B_{t_{i}^{n}}, t_{i}^{n}\right)\right] \cdot\left[B_{t_{i+1}^{n}}-B_{t_{i}^{n}}\right]
$$

exists in $P^{x}$-probability.

(b) For each $t>0$,

$$
-[g(B, \cdot), B]_{t}=\left(\int_{0}^{t} g\left(B_{s}, t-s\right) d B_{s}\right) \circ r_{t}+\int_{0}^{t} g\left(B_{s}, s\right) d B_{s}, \quad P \text {-a.s. }
$$

Now it is an easy consequence of (1) and the simple Markov property that for each $u>0$ and Lebesgue a.e. $x \in \mathbb{R}$, the process $t \mapsto f\left(B_{t}, t+u\right)$ is $P^{x}$-a.s. locally of zero quadratic variation (as in (1), but along the dyadic rationals shifted by $u$ ); from this and the finite quadratic variation of Brownian paths we deduce that $[f(B, \cdot+u), B]_{t}=0, P$-a.s., for all $t, u>0$. In view of (5) (applied to $g:(x, s) \mapsto f(x, v-t+s)$ where $v>t)$ we therefore have the antisymmetry property

$$
\left(\int_{0}^{t} f\left(B_{s}, v-t+s\right) d B_{s}\right) \circ r_{t}=-\int_{0}^{t} f\left(B_{s}, v-s\right) d B_{s}
$$

and in turn the identity

$$
Z_{t}\left(r_{t} \omega, v-t\right)=\hat{Z}_{t}(\omega, v)
$$


valid for $P$-a.e. $\omega$, for all $0<t<v$. Thus, with $\psi$ and $\varphi$ as in (4), we have

$$
\begin{aligned}
\left(\psi, Q_{t} \varphi\right) & =\int_{0}^{\infty} \int_{\mathbb{R}} \psi(x, u) P^{x}\left(Z_{t}(\cdot, u) \varphi\left(B_{t}, t+u\right)\right) d x d u \\
& =\int_{0}^{\infty} P\left(\psi\left(B_{0}, u\right) Z_{t}(\cdot, u) \varphi\left(B_{t}, t+u\right)\right) d u \\
& =\int_{0}^{\infty} P\left(\psi\left(B_{t}, u\right) Z_{t}\left(r_{t} \cdot, u\right) \varphi\left(B_{0}, t+u\right)\right) d u \\
& =\int_{t}^{\infty} P\left(\psi\left(B_{t}, v-t\right) Z_{t}\left(r_{t}, v-t\right) \varphi\left(B_{0}, v\right)\right) d v \\
& =\int_{t}^{\infty} P\left(\psi\left(B_{t}, v-t\right) \hat{Z}_{t}(\cdot, v) \varphi\left(B_{0}, v\right)\right) d v \\
& =\left(\hat{Q}_{t} \psi, \varphi\right)
\end{aligned}
$$

which proves (4).

Now (4) implies that $\left(Q_{t}\right)$ and $\left(\hat{Q}_{t}\right)$ act as strongly continuous contraction semigroups in $L^{2}(E, d x \otimes d u)$, and that they are, as such, adjoints of each other. In particular, the infinitesimal generators of these two semigroups are adjoints of each other. From this observation, (2), and (3), we deduce that

$$
\int_{0}^{\infty} \int_{\mathbb{R}} f(x, u) \psi(x, u) \frac{\partial \varphi}{\partial x}(x, u) d x d u=-\int_{0}^{\infty} \int_{\mathbb{R}} f(x, u) \varphi(x, u) \frac{\partial \psi}{\partial x}(x, u) d x d u
$$

for all smooth compactly supported functions $\psi$ and $\varphi$ on $E$. Choosing $\psi$ to be constant on a neighborhood of the support of $\varphi$, we find that

$$
\int_{0}^{\infty} \int_{\mathbb{R}} f(x, u) \frac{\partial \varphi}{\partial x}(x, u) d x d u=0
$$

which means that the distribution-sense partial derivative $\partial f / \partial x$ vanishes, so $f$ is indeed a function of its second variable alone.

The multi-dimensional version of Theorem 1 follows by an induction on the dimension; see Corollary 1 in the next section. More generally, most of the proof of Theorem 1 applies to a broad class of symmetric (i.e., reversible) Markov diffusion processes. The main difficulty in the abstract setting comes at the final step, where one would like to deduce the constancy of $f$ (as a function of the spatial variables) from the fact that certain "directional derivatives" of $f$ vanish in a weak sense. This final step can be carried out for certain diffusions in manifolds and infinite dimensional vector spaces, but the general case has proved elusive.

For more on time reversal in connection with the Cameron-Martin-Girsanov theorem (in the spirit of the proof of Theorem 1), see [1] and [2].

\section{Complements}

The following is a simple consequence of Theorem 1 .

Corollary 1. Let $\left(B_{t}^{1}, B_{t}^{2}, \ldots, B_{t}^{d}\right)_{t \geq 0}$ be d-dimensional Brownian motion starting at $\mathbf{0}$, and let $f: \mathbb{R}^{d} \times\left[0, \infty\left[\rightarrow \mathbb{R}\right.\right.$ be a continuous function. If $t \mapsto f\left(B_{t}^{1}, B_{t}^{2}, \ldots, B_{t}^{d}, t\right)$ is locally of zero quadratic variation, then $f$ is a function of $t$ alone.

Proof. Owing to the independence of the coordinates of Brownian motion, we may assume that $\left(B^{1}, B^{2}, \ldots, B^{d}\right)$ is realized on a product probability space $(\Omega, \mathcal{F}, P) \otimes$ $\left(\Omega^{\prime}, \mathcal{F}^{\prime}, P^{\prime}\right)$, such that $B^{1}$ is defined on the first factor space and $\left(B^{2}, \ldots, B^{d}\right)$ on 
the second. Given $\omega^{\prime} \in \Omega^{\prime}$, we apply Theorem 1 to the 1-dimensional Brownian motion $B^{1}$ and the continuous function $f_{\omega^{\prime}}(x, t):=f\left(x, B_{t}^{2}\left(\omega^{\prime}\right), \ldots, B_{t}^{d}\left(\omega^{\prime}\right), t\right)$. The upshot is that for $P^{\prime}$-a.e. $\omega^{\prime} \in \Omega^{\prime}, f_{\omega^{\prime}}$ does not depend on its first coordinate. By Fubini's theorem and the strict positivity of the Brownian transition density, we must have $f\left(x_{1}, x_{2}, \ldots, x_{d}, t\right)=f\left(0, x_{2}, \ldots, x_{d}, t\right)$ for all $\left(x_{1}, x_{2}, \ldots, x_{d}\right) \in \mathbb{R}^{d}$ and all $t \geq 0$. Proceeding inductively we find that $f$ does not depend on its "spatial" variables at all.

Let $B$ be a 1-dimensional Brownian motion as in section 2. As usual, we write $\theta_{t}$ for the shift operator $\left[\theta_{t} \omega\right](s)=\omega(t+s)$. A continuous additive functional (CAF) of $B$ is an $\left(\mathcal{F}_{t}\right)$-adapted real-valued process $\left(A_{t}\right)_{t \geq 0}$ such that $A_{t+s}=A_{t}+A_{s} \circ \theta_{t}$ for all $s, t \geq 0$, almost surely. From $[10, \S 4]$ we know that if a CAF $A$ is increasing (equivalently, non-negative), then it is even in the sense that $A_{t} \circ r_{t}=A_{t}, P$-a.s., for all $t>0$. (More generally, using a result of Tanaka [9], it can be shown that a CAF is even if and only if it is locally of zero quadratic variation $P$-a.s.; $c f$. [1, Thm. 2.1, Thm. 3.2]. The reader is invited to formulate a version of Theorem 2 below for general even CAFs.)

Given an increasing CAF $A$, define "space-time" processes $Y$ and $\hat{Y}$ by $Y_{t}(\omega, u)=$ $\left(B_{t}(\omega), u+A_{t}(\omega)\right)$ and

$$
\hat{Y}_{t}(\omega, u):= \begin{cases}\left(B_{t}(\omega), u-A_{t}(\omega)\right), & 0 \leq t<\tau(\omega, u) \\ \delta, & t \geq \tau(\omega, u)\end{cases}
$$

where $\tau(\omega, u):=\inf \left\{t: A_{t}(\omega) \geq u\right\}$ is the lifetime of $\hat{Y}$. Because $A$ is even, $Y$ and $\hat{Y}$ are in duality with respect to Lebesgue measure on $\mathbb{R} \times] 0, \infty[$. These facts in hand, the obvious modifications of the proof of Theorem 1 yield the following result.

Theorem 2. Let $B=\left(B_{t}\right)_{t \geq 0}$ be a 1-dimensional Brownian motion. Let $\left(A_{t}\right)_{t \geq 0}$ be an increasing $C A F$ of $B$, and let $f: \mathbb{R} \times[0, \infty[\rightarrow \mathbb{R}$ be a continuous function. If $t \mapsto f\left(B_{t}, A_{t}\right)$ is $P^{0}$-a.s. of zero quadratic variation (in the sense of (1)), then $f(x, t)=f(0, t)$ for all $x \in \mathbb{R}$ and all $t \geq 0$.

Theorem 2 implies a variant of Theorem 1 in which Brownian motion is replaced by a regular 1-dimensional diffusion. For simplicity we restrict attention to diffusions with infinite lifetime and inaccessible boundary. The proof involves a time change, which may not preserve "zero quadratic variation along the dyadic rationals." For this reason we impose a stronger "locally finite variation" condition, which is stable under time change.

We refer the reader to [4] or [6] for details on 1-dimensional diffusions, their scale functions and speed measures.

Corollary 2. Let $Y=\left(Y_{t}\right)_{t \geq 0}$ be a regular 1-dimensional diffusion on an open interval $I=] a, b\left[\subset \mathbb{R}\right.$, with fixed initial state $Y_{0}=y_{0} \in I$. Assume that $Y$ has infinite lifetime, and scale function $S$ satisfying $S(a+)=-\infty, S(b-)=+\infty$. Let $f: I \times\left[0, \infty\left[\rightarrow \mathbb{R}\right.\right.$ be continuous. If $t \mapsto f\left(Y_{t}, t\right)$ is almost surely of finite total variation on each compact interval $[0, T], T>0$, then $f$ is a function of its second argument alone.

Proof. Let $S$ (resp. $m$ ) be a scale function (resp. speed measure) for $Y$, the scale being chosen so that $S\left(y_{0}\right)=0$. The image measure $\mu:=S_{*} m$ is then a positive Radon measure on $\mathbb{R}$, with $\operatorname{supp} \mu=\mathbb{R}$. Let $B=\left(B_{t}\right)_{t \geq 0}$ denote a standard 
1-dimensional Brownian motion with $B_{0}=0$, and let $\left(L_{t}^{x}\right)_{t>0, x \in \mathbb{R}}$ be a jointly continuous version of local time for $B$. The integral $A_{t}:=\int_{\mathbb{R}} L_{t}^{x} \mu(d x)$ defines a finite strictly increasing CAF of $B$, with $\lim _{t \rightarrow \infty} A_{t}=+\infty$. The process $S^{-1}\left(B_{A^{-1}(t)}\right)$, $t \geq 0$, is equal in law to $Y$. Consequently, $t \mapsto f\left(S^{-1}\left(B_{A^{-1}(t)}\right), t\right)$ is locally of bounded variation, almost surely. Defining $g(b, a):=f\left(S^{-1}(b), a\right)$ and changing time, we see that $g\left(B_{t}, A_{t}\right)$ is locally of bounded variation. By Theorem $2, g$ is a function of $t$ alone; hence so is $f$.

\section{REFERENCES}

[1] P. J. Fitzsimmons, Even and odd continuous additive functionals, Dirichlet Forms and Stochastic Processes (Z. M. Ma, M. Röckner, J. A. Yan, eds.), (Proc. Internat. Conf. on Dirichlet Forms and Stochastic Processes, Beijing, 1993, de Gruyter, Berlin, 1995, pp. 139-154. MR 97c:60191

[2] P. J. Fitzsimmons, Drift transformations of symmetric diffusions and duality, Preprint, 1997.

[3] H. Föllmer, P. Protter and A. N. Shiryaev, Quadratic variation and an extension of Itô's formula, Bernoulli 1 (1995), 149-169. MR 96k:60121

[4] K. Itô and H. P. McKean, Jr., Diffusions Processes and their Sample Paths, Second printing, corrected, Springer-Verlag, Berlin-Heidelberg-New York, 1974. MR 49:9963

[5] F. B. Knight, On a Brownian motion problem of T. Salisbury, Canad. Math. Bull. 40 (1997), 67-71. CMP 97:11

[6] D. Revuz, D. and M. Yor, Continuous Martingales and Brownian Motion, Second edition, Springer-Verlag, Berlin, 1994. MR 95h:60072

[7] T. S. Salisbury, An increasing diffusion, Seminar on Stochastic Processes, 1984 (E. Çinlar, K. L. Chung, R. K. Getoor, eds.), Birkhäuser, Boston, 1986, pp. 173-194. MR 88k:60138

[8] M. J. Sharpe, General Theory of Markov Processes, Academic Press, San Diego, 1988. MR 89m:60169

[9] H. Tanaka, Note on continuous additive functionals of the 1-dimensional Brownian path, Z. Wahrscheinlichkeitstheorie und verw. Gebiete 1 (1962/63), 251-257. MR 29:6559

[10] J. B. Walsh, Markov processes and their functionals in duality, Z. Wahrscheinlichkeitstheorie und verw. Gebiete 24 (1972), 229-246. MR 48:7398

Department of Mathematics, University of California San Diego, 9500 Gilman Drive, La Jolla, California 92093-0112

E-mail address: pfitz@euclid.ucsd.edu 